\title{
Correction to: Flash Crowd Management in Beyond 5G Systems
}

\author{
Valentin Rakovic, Hristijan Gjoreski, Marija Poposka, \\ Daniel Denkovski, and Liljana Gavrilovska
}

\section{Correction to: \\ Chapter "Flash Crowd Management in Beyond 5G Systems" in: D. Perakovic and L. Knapcikova (Eds.): Future Access Enablers for Ubiquitous and Intelligent Infrastructures, LNICST 382, https://doi.org/10.1007/978-3-030-78459-1_4}

The original version of this book was inadvertently published with the first author surname incorrect, which has now been corrected. 